\title{
Glymphatic fluid transport controls paravascular clearance of AAV vectors from the brain
}

\author{
Giridhar Murlidharan, ${ }^{1,2}$ Andrew Crowther, ${ }^{3,5}$ Rebecca A. Reardon, ${ }^{2}$ Juan Song, ${ }^{4,5}$ \\ and Aravind Asokan ${ }^{2,6,7}$ \\ ${ }^{1}$ Curriculum in Genetics and Molecular Biology, ${ }^{2}$ Cene Therapy Center, ${ }^{3}$ Neurobiology Curriculum, ${ }^{4}$ Department of \\ Pharmacology, ${ }^{5}$ University of North Carolina Neuroscience Center, ${ }^{6}$ Department of Cenetics, and ${ }^{7}$ Department of \\ Biochemistry and Biophysics, The University of North Carolina at Chapel Hill, Chapel Hill, North Carolina, USA.
}

\begin{abstract}
Adeno-associated viruses (AAV) are currently being evaluated in clinical trials for gene therapy of CNS disorders. However, host factors that influence the spread, clearance, and transduction efficiency of AAV vectors in the brain are not well understood. Recent studies have demonstrated that fluid flow mediated by aquaporin-4 (AQP4) channels located on astroglial end feet is essential for exchange of solutes between interstitial and cerebrospinal fluid. This phenomenon, which is essential for interstitial clearance of solutes from the CNS, has been termed glial-associated lymphatic transport or glymphatic transport. In the current study, we demonstrate that glymphatic transport profoundly affects various aspects of AAV gene transfer in the CNS. Altered localization of AQP4 in aged mouse brains correlated with significantly increased retention of AAV vectors in the parenchyma and reduced systemic leakage following ventricular administration. We observed a similar increase in AAV retention and transgene expression upon i.c.v. administration in AQP4-1mice. Consistent with this observation, fluorophore-labeled AAV vectors showed markedly reduced flux from the ventricles of AQP4 ${ }^{-/-}$mice compared with WT mice. These results were further corroborated by reduced AAV clearance from the AQP4-null brain, as demonstrated by reduced transgene expression and vector genome accumulation in systemic organs. We postulate that deregulation of glymphatic transport in aged and diseased brains could markedly affect the parenchymal spread, clearance, and gene transfer efficiency of AAV vectors. Assessment of biomarkers that report the kinetics of CSF flux in prospective gene therapy patients might inform variable treatment outcomes and guide future clinical trial design.
\end{abstract}

Conflict of interest: A. Asokan is an inventor on patents (PCT/ US2016/013460; WO/2014/144229; Wo/2012/109570; WO/2010/093784; wo/2007/089632) licensed out by UNC-Chapel Hill and a co-founder at StrideBio.

Submitted: April 12, 2016 Accepted: August 2, 2016 Published: September 8, 2016

Reference information: JCI Insight. 2016;1(14):e88034 doi:10.1172/ji.insight.88034.

\section{Introduction}

Recombinant adeno-associated viral (AAV) vectors are currently being evaluated in phase $1 / 2$ gene therapy clinical trials for treating diseases, such as spinal muscular atrophy (NCT02122952), giant axonal neuropathy (NCT02362438), Parkinson disease (NCT00229736), Alzheimer disease (NCT00017940), and lysosomal storage disorders, such as Sanfilippo syndrome (NCT01474343). While these early studies corroborate the safety of AAV vectors in the CNS, the efficiency of delivery and gene expression in different indications has been variable. Importantly, the potential mechanisms underlying such outcomes are not well understood. For instance, a 10-year follow-up study of postmortem brain tissue obtained from patients with Alzheimer disease treated with AAV2/nerve growth factor (NGF) demonstrated axonal sprouting toward the NGF expression site (1). In contrast, gene therapy clinical trials for Parkinson disease have highlighted low transduction efficiency in certain regions, such as the putamen, and decreased axonal transport in pathological brain tissue as potential challenges (2). Thus, ongoing and future clinical evaluation of AAV vectors for treating neurodegenerative disorders could benefit from mechanistic studies in predictive in vivo systems that accurately depict physiological and biochemical landscape of the human brain.

In this regard, techniques, such as convection-enhanced delivery of AAV vectors, and optimal conditions for vector infusion into the cerebrospinal fluid (CSF) through intrathecal administration are being explored to target a larger brain volume (3). Such methods under development could be vastly improved by a better understanding of the mechanisms that dictate AAV transport in the brain. In this regard, it is well established 
that the brain lacks conventional lymphatic vasculature. Instead, a combination of CSF and water moves via subarachnoid spaces and paravascular compartments to drain extracellular depositions like metabolites and proteins (4-6). At a mechanistic level, CSF engages in direct exchange of biomaterials with proximal CNS tissue via subarachnoid ducts $(5,7)$. Further, interstitial deposits are constantly shunted across the CNS tissue due to transparenchymal water transport. Specifically, vascular pulsation generates convective currents of water from aquaporin-4 (AQP4) channels located on astroglial end feet along paravascular compartments (4). Such currents facilitate the drainage of solutes accumulating in the CNS into proximal paravenous ducts and their subsequent clearance into peripheral lymphatics $(4,8)$. This paravascular clearance mechanism via CSF flux constitutes the "glymphatic" clearance (4, 8-10). Although the biology of several AAV serotypes and their interactions with cell surface receptors are well understood, potential host factors affecting AAV transport within the brain remain largely unknown $(11,12)$. Thus, there is a clear need to better understand the checkpoints that govern AAV vector transport, transduction efficiency, and clearance in the CNS.

In the current study, we hypothesized that transparenchymal CSF flux via glymphatic clearance has profound implications for AAV transport in the brain. At the molecular level, an expanding body of evidence suggests that this paravascular fluid flux is regulated by AQP4 channels that line the astrocytic end feet $(4,8,13,14)$. Based on this rationale, we specifically evaluated the effect of abnormal AQP4 expression on CNS transport and gene transfer properties of recombinant AAV vectors in vivo. We chose AAV serotype 9 (AAV9), which is currently being utilized in different clinical trials focused on treating neurological disorders, as a model capsid for our studies. Specifically, we assessed AAV transport in mice displaying (a) age-dependent dysregulation of AQP4 and (b) genetic knockout of AQP4. Our studies demonstrate that AQP4-mediated CSF flux profoundly influences AAV transport in the brain.

\section{Results}

Increased retention of $A A V$ vectors in the aging mouse $C N S$. The aging brain is susceptible to increased parenchymal deposition of metabolic wastes (4). We performed intracranial injections of AAV9 into juvenile (3-month-old) and aged (>18-month-old) mouse brains. In order to visualize viral particles within the brain, equal mixtures of Alexa 647-labeled AAV9 vectors $\left(1.75 \times 10^{9}\right.$ vector genomes $\left.[\mathrm{vg}] / \mathrm{mouse}\right)$ and (0.5\%) Alexa $488-$ labeled dextran (10-kDa soluble tracer) were coadministered into the ventricles. At 45 minutes after injection, mice were rapidly anesthetized and brains were harvested after cardiac perfusions with PBS followed by $4 \%$ paraformaldehyde (PFA). Post-fixed brains were sectioned on a vibratome, and a Zeiss 700 confocal microscope was used to obtain whole-brain images by stitching multiple ventricular slices. We observed restricted fluorescence (red) from AAV9 vector particles at close proximity to the ventricular site of administration in the juvenile mouse cohort (Figure 1A). On the other hand, identical injections performed on aged mice resulted in markedly enhanced fluorescence (red) in deeper anatomical regions across the brain parenchyma (Figure 1B). A similar increase in fluorescence signal (green) was also observed from dextran tracer injections in aged mice (Figure 1B) as compared with juvenile mice (Figure 1A). It is noteworthy to mention that we also observed increased fluorescence from AAV9 vectors (red) and dextran tracer (green) along the needle track and within parenchymal tissue surrounding the lateral ventricles (LV) (Figure 1, A and B). It is likely that the injection mixture permeated the brain parenchyma during stereotaxic surgery. More importantly, these observations indicate that the increase in parenchymal accumulation within the aged brain is not specific to AAV vectors.

To further demonstrate AAV accumulation in the aging brain, we generated high-magnification confocal micrographs of 6 brain regions: i.e., LV, corpus callosum (CC), cortex (CT), piriform cortex (PCT), thalamus (THL), and hypothalamus (HTL). Fluorescent signal from AAV vectors and dextran tracers was restricted to LV and CC regions in the juvenile mouse brain (Figure 1C). However, fluorescent signal was observed in all 6 labeled anatomical regions in the aging mouse brain (Figure 1D). No significant difference in AAV fluorescence in the 2 groups was observed in the area surrounding the LV site of injection (Figure $1 \mathrm{E}$, region 1). However, quantitation of fluorescence intensities elicited by AAV9 vectors showed a significant increase in CC, CT, PCT, THL, and HTL regions of aged mouse brains when compared with the juvenile mice (Figure 1, E and F, regions 2-6). A similar overall pattern was observed with dextran tracers in the aging mouse brain (Figure 1, $\mathrm{G}$ and $\mathrm{H}$ ).

Altered AQP4 localization correlates with increased AAV deposits and reduced systemic AAV leakage in aging mice. Loss of perivascular AQP4 polarity is one of the histological hallmarks that indicate impairment of CSF penetration through the brain parenchyma (14). To assess whether altered localization of AQP4 plays 
A
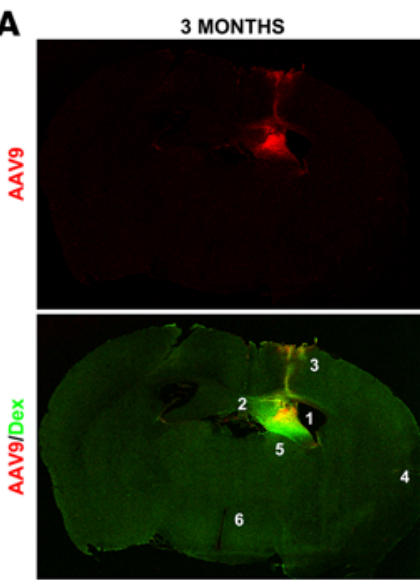

B
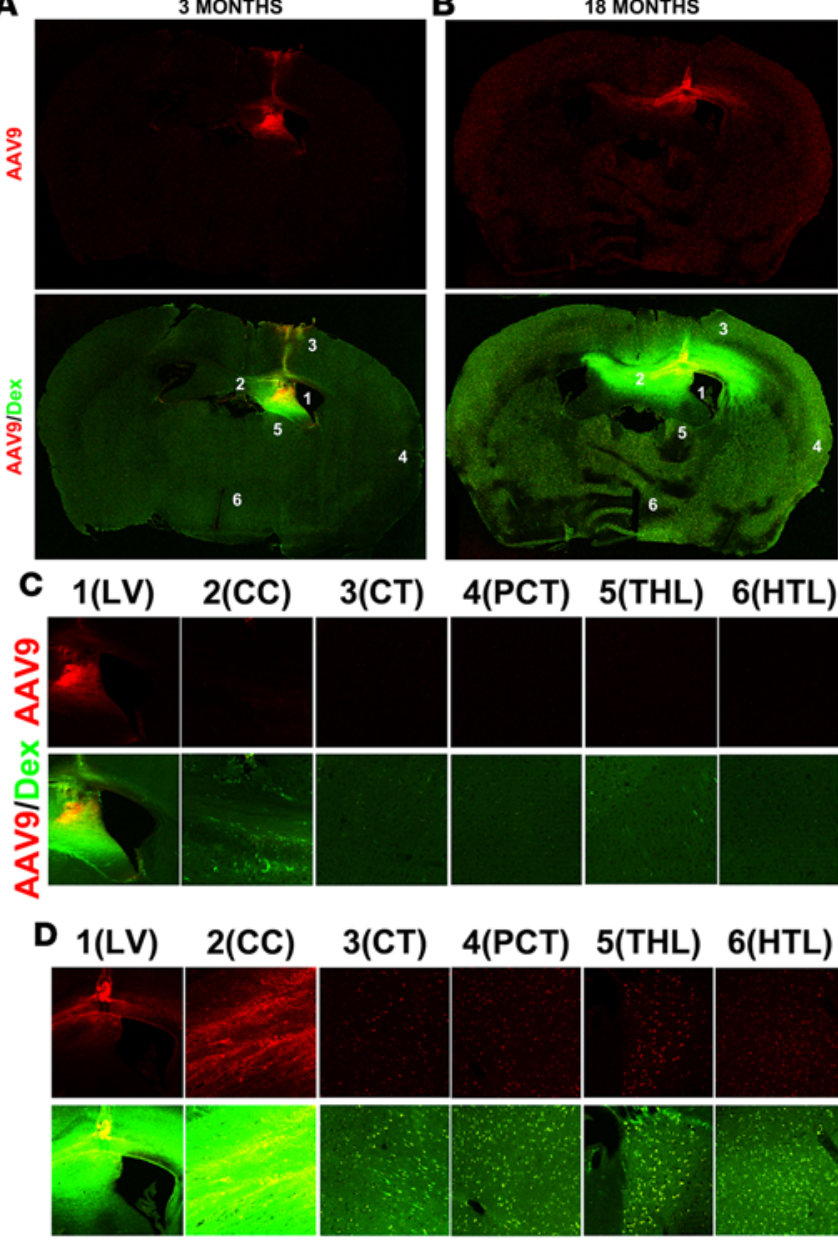

E

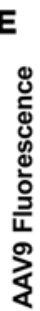

G

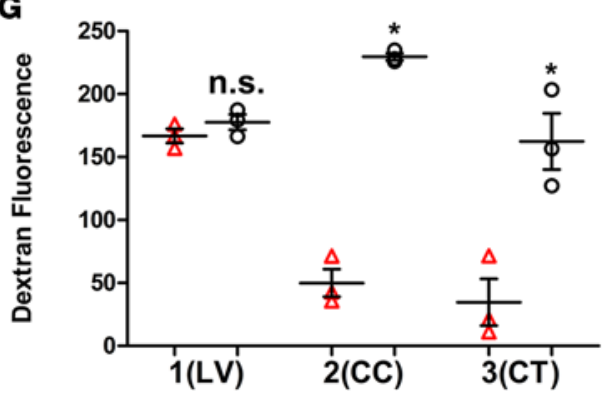

Figure 1. Comparison of AAV vector accumulation within juvenile and aging mouse brains. Three-monthold and eighteen-month-old mice were injected with equal viral titers $\left(1.75 \times 10^{9} \mathrm{vg}\right.$ per animal) of Alexa 647-tagged fluorescent AAV9 vectors mixed with $0.5 \%$ Alexa 488-tagged fluorescent $10-\mathrm{kD}$ a dextran tracer (5 $\mathrm{mg} / \mathrm{ml}$ ) into the left lateral ventricle. 45 minutes after injections, the mice were anesthetized and sacrificed for organ harvests. Paraformaldehyde-fixed brains were sectioned on a vibratome, and a Zeiss 700 confocal microscopy was used to generate fluorescence images of the mouse brains. (A and B) Representative stitched confocal images of coronal sections containing the lateral ventricular site of injection. The top row shows accumulation of fluorophore-labeled AAVg (red). The bottom row shows merge of AAV (red) and dextran (green) fluorescent signals. Additionally, 6 brain regions have been numbered 1-6, i.e., lateral ventricles (LV), corpus callosum (CC), cortex (CT), piriform cortex (PCT), thalamus (THL), and hypothalamus (HTL), as positional cues to higher magnification images below. (C and D) Higher-magnification confocal micrographs show regions of interest in juvenile (C) and aging (D) mouse cohorts. Images show differences in fluorescence signal due to AAV (red) and AAV/dextran merge (red/green). Original magnification $\times 7.5$. Quantitation of fluorescence intensities from AAV (E and $\mathbf{F}$ ) and dextran ( $\mathbf{G}$ and H) channels within regions $1-6$ (mean \pm SEM). $P$ values were calculated by unpaired, 2-tailed Student's $t$ test. ${ }^{*} P<0.05$. All experiments were conducted in triplicate, and representative images are shown.

a role in accumulation of AAV vectors within the aged mouse brain, we analyzed AAV capsid accumulation and AQP4 expression profiles in juvenile and aging mouse brains. Fluorescently labeled AAV9 particles were injected through the intra-CSF route in juvenile (3-month-old) and aged (18-month-old) mouse brains. At 45 minutes after administration, animals were sacrificed and post-fixed in 4\% PFA. High-mag- 

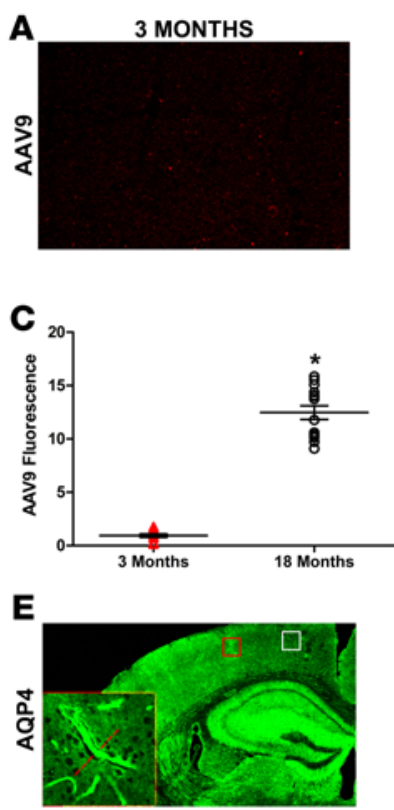

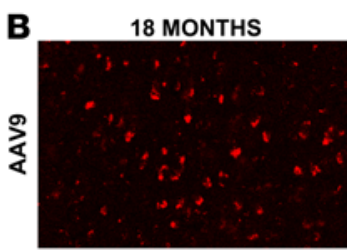

D
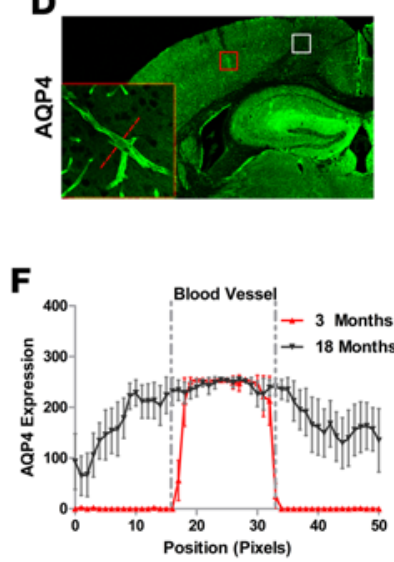

Figure 2. Aged mice display abnormal AAV accumulation and altered AQP4 localization in the brain. (A and B) Comparison of accumulation of fluorophore-labeled AAV9 vector particles (red) 45 minutes after i.c.v. injections in the 3-month-old and 18-month-old mouse brain. (C) Quantitation of fluorescence intensities due to AAV9 accumulation within cortical regions of 3 -month-old and 18 -month-old mice (mean \pm SEM). $P$ values were calculated by unpaired, 2 -tailed Student's $t$ test. ${ }^{*} P<0.05$. ( $\mathbf{D}$ and $\mathbf{E}$ ) Three-month-old and eighteen-month-old mouse brains injected with fluorescent AAV9 vectors (Figure 1) were immunostained for AQP4 expression (green). Red boxes indicate positions of large blood vessels (arterioles) shown in higher magnification within the insets on the left. White boxes indicate cortical regions in which AAV accumulation was compared. (F) Fluorescence intensity (green) of AQP4 expression was measured as a function of distance (in pixels) across 4 to 5 large blood vessels (red boxes within) per mouse (red dotted lines, insets within $\mathbf{D}$ and $\mathbf{E}$ ). Error bars on the graph represent the $95 \%$ confidence interval of fluorescence intensity calculated at every pixel position across the blood vessel. All experiments were conducted in triplicate, and representative images are shown. (A and $\mathbf{B}$ ) are $\times 10$ magnification of ( $\mathbf{D}$ and $\mathbf{E})$

nification confocal micrographs of cortical regions (Figure 2, D and E) revealed that the fluorescent signal from AAV9 particles (red) was strikingly elevated and appeared as deposits in the aged mouse brain (Figure 2B) as compared with the juvenile cohort (Figure 2A). Quantitation of this phenomenon shows a significant increase in fluorescence signal associated with AAV capsids within cortical regions of aged mouse brains compared with juvenile mice (Figure 2C).

Next, to compare perivascular AQP4 polarity, we generated high-magnification confocal micrographs of AQP4 expression within juvenile and aged mouse brains. Specifically, we compared AQP4 localization on penetrating large blood vessels (arterioles) within the cortex (Figure 2, D and E, red boxes and insets). When compared with juvenile mice (Figure 2D, inset), we observed a striking increase in AQP4 expression (green) immediately surrounding the blood vessels in the aged mice (Figure 2E, inset). These results are consistent with loss of AQP4 polarity, as reported earlier in the literature (14). To quantify this phenomenon, we measured fluorescence intensities in pixel positions across the blood vessel walls (Figure 2, D and $\mathrm{E}$, red dotted lines in insets). In juvenile mice, we observed a noticeable increase in AQP4 expression within the blood vessel architecture with low, background level fluorescence signal in the surrounding tissue (Figure 2F). However, AQP4 expression was mislocalized and broadly distributed in areas adjacent to the blood vessels of the aging mice (Figure $2 \mathrm{~F}$ ).

To further understand whether increased AAV deposition in the CNS affects systemic leakage of AAV vectors, we evaluated the biodistribution of AAV9 vg within juvenile and aging mice. At 45 minutes after intra-CSF administration, quantitation of vg copy numbers in systemic organs (normalized to the brain) was carried out. We observed significant reduction in vg uptake in hearts $(\sim 165$-fold) and livers $(\sim 17$ fold) of aged mice when compared with juvenile mice (Figure 3). Overall, these results suggest a potential correlation between altered AQP4 expression patterns and increased parenchymal accumulation of AAV vectors, resulting in reduced systemic vector leakage in aged mice.

$A A V$ transduction is markedly altered in the brains of $A Q P 4^{-1}$ mice. To further delineate the role of AQP4 in other aspects of AAV-mediated CNS gene transfer, we utilized the AQP4 knockout ( $\mathrm{AQP} 4^{--}$) mouse model (15). Complete loss of AQP4 expression was confirmed by immunohistochemical analysis of WT and $\mathrm{AQP}^{-/-}$mouse brain sections (Supplemental Figure 1; supplemental material available online with this article; doi:10.1172/jci.insight.88034DS1). We first compared transduction profiles of AAV9 vectors injected unilaterally into the ventricular CSF space (i.c.v. injections). Specifically, we injected neonatal P0 mice with AAV9 vectors packaging a self-complementary GFP (ScGFP) cassette driven by a chicken $\beta$ hybrid (CBh) promoter. At 2 weeks after administration, $50-\mu \mathrm{m}$ vibratome brain sections were subject to diaminobenzidine (DAB) immunohistochemistry to visualize transgene (GFP) expression. AAV9 injections in WT mice resulted in broad GFP 


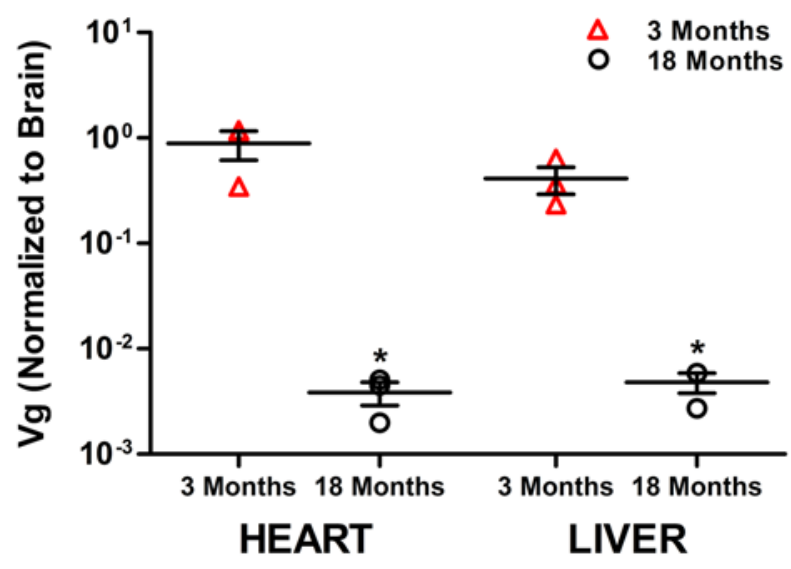

Figure 3. Biodistribution of AAV vectors in systemic organs following intraCSF administration in juvenile and aging mouse brains. Three-month-old and eighteen-month-old mice were injected with equal viral titers of AAV9 vectors (Figure 1) and sacrificed 45 minutes after intra-CSF administrations. Vector genome (vg) copy numbers within systemic organs, hearts and livers (normalized to vg in the brain), of juvenile and aging mice are shown. The vg copy per host genome (vg/cell) was determined by quantitative PCR of extracted genomic DNA and normalized to the number of copies of the mouse lamin $\beta$ gene (mean $\pm \mathrm{SEM})$. $P$ values were calculated by unpaired, 2-tailed Student's $t$ test. ${ }^{*} P<0.05$. All experiments were conducted in triplicate.

expression across the brain parenchyma (Figure 4A). Interestingly, i.c.v. administration of AAV9 in AQP4 ${ }^{-/-}$ mice resulted in dramatically enhanced GFP expression in the CNS (Figure 4A). Specifically, we observed a substantial increase in $\mathrm{GFP}^{+}$cells in representative regions of the contralateral hemisphere after unilateral i.c.v. injections in the $\mathrm{AQP}^{-/-}$mice (Figure $4 \mathrm{~A}$, boxes and insets). We measured pixel intensities to quantify GFP expression across different brain regions, specifically, the motor, somatosensory, auditory, and piriform cortices as well as the striatum and hippocampus. A significant increase in pixel intensities within the aforementioned regions in $\mathrm{AQP} 4^{-/-}$mouse brain was observed when compared with the WT brain (Figure 4B). At a mechanistic level, AAV vectors administered directly into the CSF are likely subject to rapid clearance. Reduced clearance of interstitial solutes due to lack of AQP4 function could result in enhanced retention of AAV vectors and consequently improved gene expression. Taken together, these results can possibly be explained by severely impaired parenchymal transport and CSF clearance of AAV vectors in $\mathrm{AQP} 4^{-/-}$mouse brains.

$A A V$ vectors display increased retention within the brains of $A Q P 4^{-1-}$ mice. To demonstrate a direct correlation between AQP4-mediated CSF flux and AAV transport in the brain, we compared the distribution of fluorescently labeled AAV vectors within WT and AQP4 ${ }^{-/-}$mouse brains. Specifically, we injected equal titers $\left(1.75 \times 10^{9} \mathrm{vg} / \mathrm{mouse}\right)$ of Alexa $647-$ labeled AAV9 mixed with equimolar concentrations $(0.5 \%)$ of Alexa 488-labeled $10-\mathrm{kDa}$ dextran tracer into neonatal (P0) mouse brains. We then

A

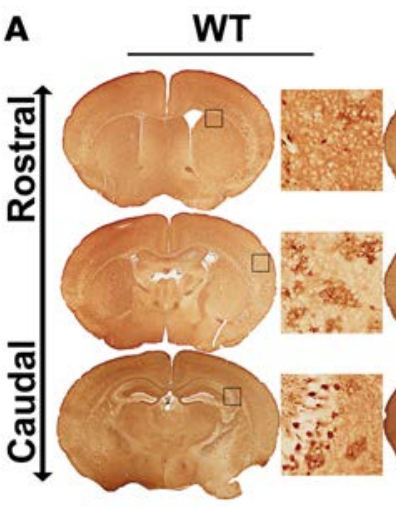

B

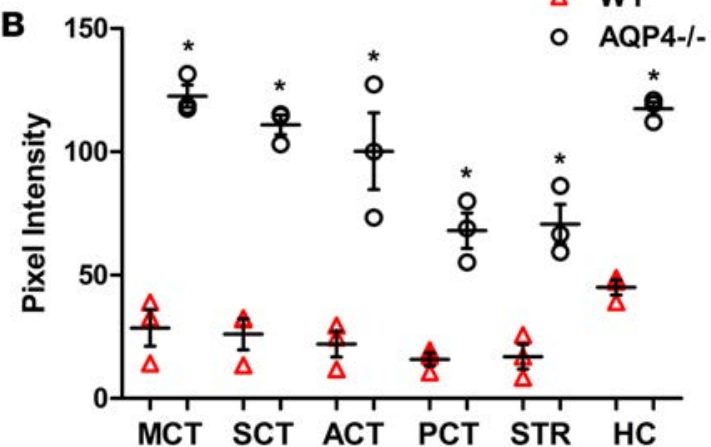

utilized the histograph function (Zen Black image analysis software) on wholebrain confocal stitched whole-brain confocal images to quantify the absolute fluorescent signal from AAV particles or the dextran tracer (Figure 5, A and B, top row). Briefly, white peaks on the surface of coronal brain sections indicate positions of fluorescent AAV9 vector particles or dextran. Unilateral intra-CSF administration of AAV9 in WT mice resulted in widespread distribution of fluorescent peak signals distributed across both hemispheres of the brain (Figure $5 \mathrm{~A}$, top row). A similar pattern was observed across the brain parenchyma in the case of fluorescent dextran tracer (Figure 5B, top row). In contrast, intra-CSF

Figure 4. Comparison of AAV transduction efficiency following intra-CSF administration in WT and AQP4 ${ }^{-/-}$mouse brains. PO mice were injected with equal viral titers ( $3.5 \times 10^{9} \mathrm{vg}$ per animal) of AAV9 packaging the self-complementary GFP transgene driven by the hybrid chicken $\beta$ actin promoter (CBh) into the left lateral ventricle. Two weeks after vector administration, the mice were sacrificed and the brains were harvested, post-fixed, and vibratome sectioned. (A) Diaminobenzidine (DAB) immunohistochemistry was used to visualize GFP expression (brown) within WT and AQP4 ${ }^{-1-}$ mouse brains. Boxes indicate the positions of higher-magnification images shown adjacent to the stitched whole-brain confocal images. Original magnification, $\times 10$. (B) Quantitation of pixel intensities from GFP expression within motor cortex (MCT), somatosensory cortex (SCT), auditory cortex (ACT), piriform cortex (PCT), striatum (STR), and hippocampal (HC) regions of WT (red) and AQP4-/- (gray) mice injected with AAV 9 vectors (mean \pm SEM). $P$ values were calculated by unpaired, 2-tailed Student's $t$ test. ${ }^{*} P<0.05$. All experiments were conducted in triplicate, and representative images are shown. 
A
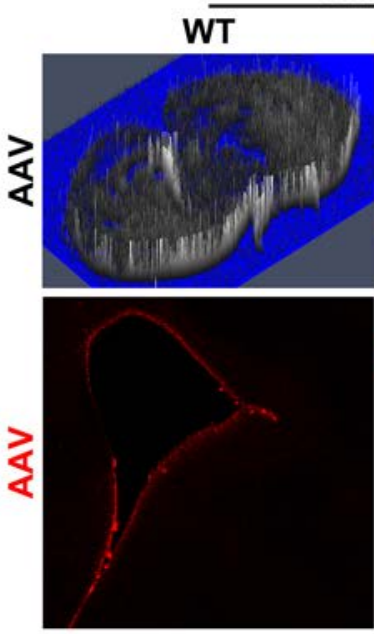

C

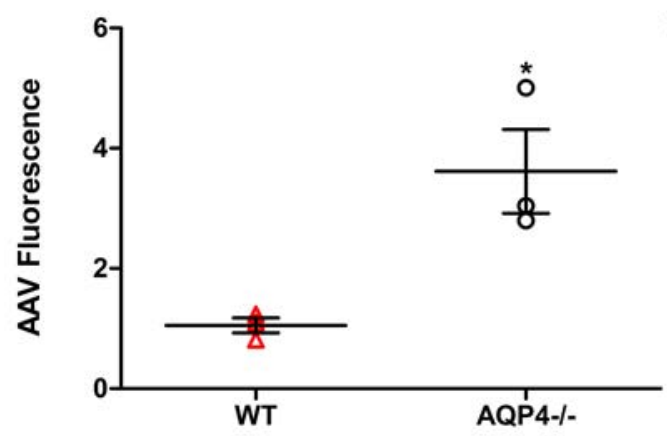

AQP4-/-
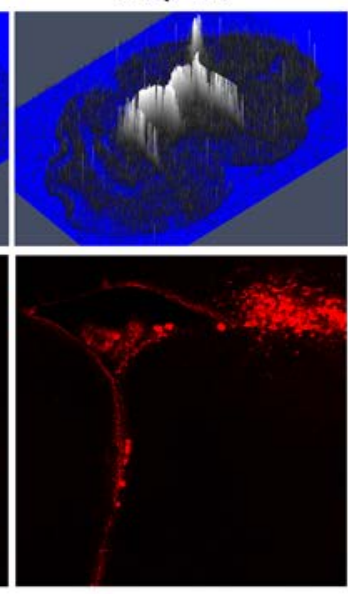

B

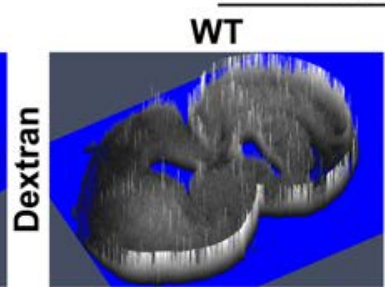

Dextran
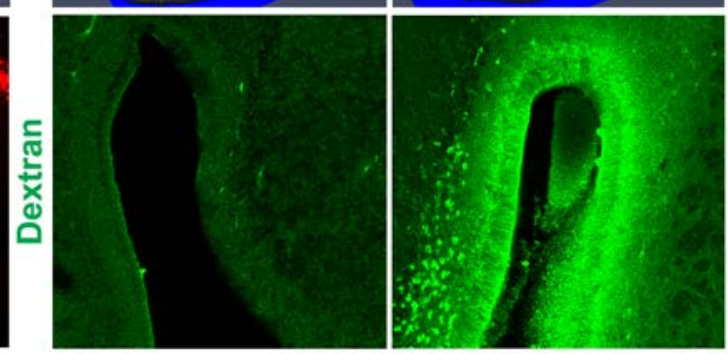

D

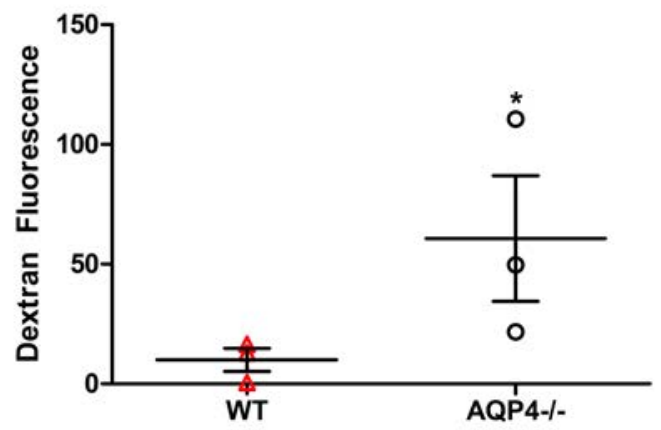

Figure 5. Comparison of AAV vector spread within WT and AQP4 ${ }^{-/-}$brains. PO WT and AQP4 ${ }^{-/-}$mice were injected with equal viral titers $\left(1.75 \times 10^{9} \mathrm{vg}\right.$ per animal) of Alexa 647-tagged fluorescent AAV9 vectors mixed with $0.5 \%$ Alexa 488 -tagged fluorescent 10-kDa dextran tracer $(5 \mathrm{mg} / \mathrm{ml})$ into the left lateral ventricle. 45 minutes after injection, the mouse pups were anesthetized and sacrificed for organ harvests. Paraformaldehyde-fixed brains were sectioned on a vibratome, and a Zeiss 700 confocal microscopy was used to generate fluorescence images of the mouse brains. (A and B) Histograph functionality (ZEN black image analysis software for Zeiss 700 laser scanning microscope) was across stitched whole-brain confocal images of $A A V+d e x t r a n$ co-injected mouse brains. White peaks within the histographs represent positions of fluorescent AAV or dextran particles within mouse brains. The bottom row shows higher-magnification fluorescence images of the lateral ventricles, with differential accumulation of AAV vectors (red) and dextran tracers (green) at the site of injection in WT and AQP4 ${ }^{-/-}$mice. Top row, original magnification $\times 20$; bottom row, original magnification $\times 4$. (C and D) Quantitation of fluorescence intensities at the lateral ventricular and subventricular sites of administration of AAV9 (C) and dextran (D) into WT and AQP4 ${ }^{-/-}$mouse brains (mean \pm SEM). P values were calculated by unpaired, 2-tailed Student's $t$ test. ${ }^{*} P<0.05$. All experiments were conducted in triplicate, and representative images are shown.

administration in $\mathrm{AQP} 4^{-/-}$mice resulted in a localized concentration of fluorescent signal from AAV vectors within the immediate vicinity of the ventricular site of injection (Figure 5A, top row). A similar reduction in spread and increased ventricular retention was observed in case of dextran tracer in $\mathrm{AQP} 4^{-/}$mice (Figure $5 \mathrm{~B}$, top row). High-magnification confocal micrographs of the LV revealed increased fluorescence corresponding to both AAV capsids and dextran tracer in the region surrounding the ventricles of $\mathrm{AQP} 4^{-/}$mice compared with WT mice (Figure 5, A and B, bottom row). Quantitation of fluorescence signal at the ventricular site of administration showed significantly increased retention of AAV particles ( $\sim 3$-fold) and dextran ( $\sim 6$-fold) in the AQP4 $4^{-/}$mouse brain as compared with WT mice (Figure 5, C and D). These results strongly support the notion that AQP4-mediated CSF flux is a critical host factor that determines AAV transport within the brain.

AQP4-mediated CSF flux drives systemic leakage of AAV vectors into peripheral organs. AQP4-mediated fluid flux within the brain is known to promote the drainage of solutes into peripheral lymphatics and, subsequently, the systemic blood circulation $(5,7)$. Within the framework of the current study, it is well known from preclinical studies that AAV9 capsids are prone to systemic leakage and tend to accumulate within organs such as the heart, liver, and spleen following CNS administration (16-18). To understand whether these attributes are affected when AQP4 function and CSF flux are impaired, we evaluated the systemic 
A

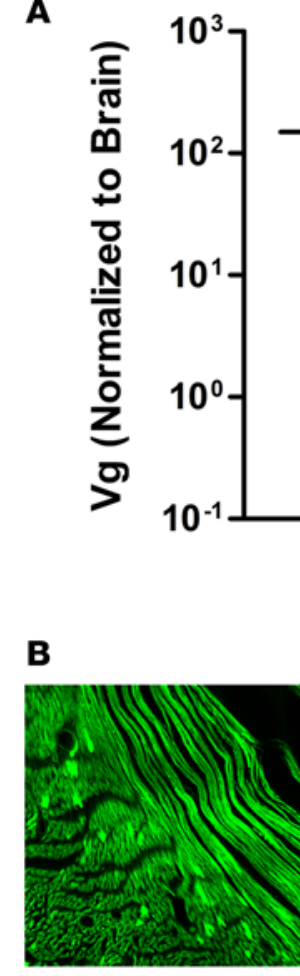

WT

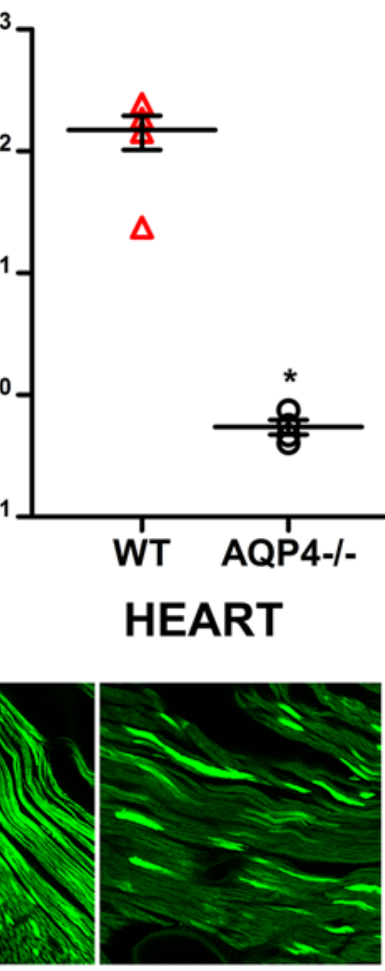

AQP4-/-

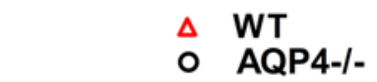

○ AQP4-/-
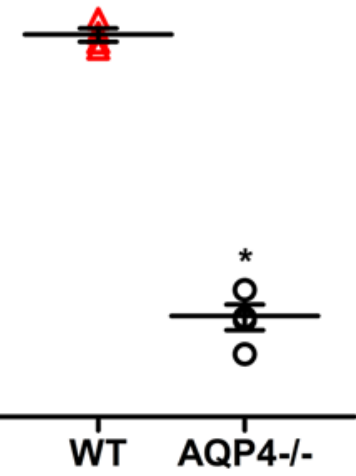

LIVER

Figure 6. Transduction and biodistribution of AAV vectors in systemic organs following intra-CSF administration in WT and AQP4-/- mice. PO WT or AQP4 ${ }^{-/-}$mice were injected with equal viral titers $\left(3.5 \times 10^{9} \mathrm{vg}\right.$ per animal) of AAVg packaging the luciferase or the self-complementary GFP transgene driven by chicken $\beta$ actin (CBA) or the hybrid chicken $\beta$ actin promoter (CBh), respectively, into the left lateral ventricle. (A) For biodistribution studies, mice were sacrificed 3 days after AAV9-CBA-Luc administration. Vector genome (vg) copy numbers within the hearts and livers of WT and AQP4 ${ }^{-/-}$mice i.c.V. injected with AAV9 vectors (normalized to vg in the brain). The vg copy per host genome (vg/cell) was determined by quantitative PCR of extracted genomic DNA and normalized to the number of copies of the mouse lamin $\beta$ gene. (B) Two weeks after AAV9-CBh-ScGFP administration, mice were sacrificed and peripheral organs were harvested, post-fixed, and vibratome sectioned. Confocal micrographs showing GFP expression (green) in systemic organs, heart and liver, following brain injections of AAV9 vectors (mean \pm SEM). Original magnification, $\times 20$. $P$ values were calculated by unpaired, 2 -tailed Student's $t$ test. ${ }^{*} P<0.05$. All experiments were conducted in quadruplicate, and representative images are shown.

biodistribution and transduction profile of AAV9 vectors administered through the intra-CSF route into WT and AQP4 ${ }^{-/}$mice. At 3 days after administration, quantitation of vg copy numbers in systemic organs revealed extensive uptake ( $\sim 150$-fold higher compared with the brain) in the hearts and livers of WT mice compared with AQP4 ${ }^{-/-}$counterparts (Figure 6A). These results were further corroborated by robust GFP expression in cardiac and liver tissues of WT mice compared with AQP4 $4^{-/}$mice 2 weeks after injections (Figure 6B). Thus, AQP4-mediated CSF flux appears to be the driving force behind the clearance of AAV vectors administered into the brain and, in turn, profoundly influences their accumulation in systemic organs such as the heart and liver.

\section{Discussion}

Recombinant AAV vectors are being evaluated as clinical vector candidates for CNS disorders such as Alzheimer disease, Parkinson disease, and frontotemporal dementia (19, 20). A common hallmark of such diseases is the parenchymal accumulation of misfolded proteins and erroneous metabolic byproducts such as $\alpha$-synuclein, hyperphosphorylated $\tau$, etc. It is well known that aging brains are more susceptible to such depositions of senile plaques $(21,22)$. Correspondingly, these neurocognitive disorders are more prevalent among the geriatric population (23). In the aging mouse brain $(>18$ months), both glymphatic CSF flux and transparenchymal solute clearance have been demonstrated to be severely impaired (14). 
In the current study, we first hypothesized that CSF flux plays a major role in retention of AAV vectors within the CNS. Our results confirm that accumulation of AAV vectors increases dramatically in the aging mouse brain. These observations appear to correlate with mislocalized expression of expression of AQP4 channels lining the surface of the major blood vessels (arteries and veins) and microvasculature (capillaries) in the brain. Moreover, our results are consistent with the notion that dysregulated AQP4 function affects interstitial fluid and solute clearance from the CNS in conditions such as Alzheimer disease, aging, and dementia $(9,10,13,14,24)$. Together with clinical studies in patients with Parkinson or Alzheimer disease $(1,2)$, our observations of AAV vector transport in aged mouse brains indicate a potential link between AQP4 function and CNS retention/CSF clearance of viral vectors. These factors, along with others that affect gene expression (2), highlight the need to better understand AAV transport in the aging population and patients with neurodegenerative disease. Although beyond the scope of the current study, additional experiments evaluating alternative routes of AAV vector administration in AQP4 ${ }^{-/-}$mice as well as aged mice, such as intracisternal, intraparenchymal, and intrathecal injections, could provide further mechanistic insight. Specifically, such studies would provide a more comprehensive understanding of the effect of glymphatic flux in different CSF compartments on CNS transport of different AAV serotypes and isolates.

To directly demonstrate a correlation between AQP4 function and AAV transport in the brain, we compared WT mice with an AQP4 knockout mouse model $\left(\mathrm{AQP}^{-/}{ }^{-}\right)(15)$. These studies led to several critical findings with regard to AAV transport in the CNS. First, a hallmark feature of $\mathrm{AQP} 4^{-/}$mice is reduced glymphatic water transport and slower clearance of parenchymal solutes such as amyloid- $\beta$ plaques from the CNS (4). Consistent with these studies, we observed restricted spread and increased retention of AAV particles within aged mice as well as AQP4 ${ }^{-/}$mice. Thus, mislocalization and/or decreased expression of AQP4 appear to adversely affect AAV transport within the brain. Paradoxically, intra-CSF administration of AAV vectors in $\mathrm{AQP}^{-/-}$brains resulted in substantially increased transgene expression, likely due to increased residence time in the CSF. The latter observation suggests that rapid turnover of CSF due to AQP4-mediated water transport in the brain is a critical host factor restricting AAV transduction in the CNS $(8,25)$. Additional optimization of AAV vector infusion rates into the CNS will likely provide further insight into such mechanisms. An important consideration is whether these findings might have any translational benefit in prospective patients. For instance, models of traumatic brain injury (TBI) show significant loss $(\sim 60 \%)$ in AQP4-mediated glymphatic water transport (10). Correspondingly, it is well established that the risk of developing neurodegenerative disorders stemming from parenchymal aggregations of neurofibrillary tangles, such as Alzheimer disease or dementia, are significantly higher in the post-TBI demographic (24, 26-29). Thus, an intriguing possibility is that AAV vectors administered in a post-TBI animal model or patient cohort might display increased retention within the CNS. It is also important to note that other factors, such as neurodegeneration and altered axonal transport, could affect AAV transport as well as gene expression profiles in aged, traumatized, or diseased brain tissue. For, instance, it is well known that AAV9 successfully employs bidirectional axonal transport in both retrograde and anterograde directions to travel long distances within the brain (30-33). Further studies delineating the relationship among receptor usage, axonal transport, and CSF flux will be essential toward developing a complete understanding of AAV transport in the brain.

Further, our studies strongly support the notion that AQP4-mediated CSF flux drives systemic leakage and biodistribution of AAV vectors administered into the CNS. As mentioned earlier, preclinical studies with several naturally occurring AAV strains have established that CNS administration through intracranial or intrathecal routes often results in vector sequestration within peripheral organs such as the liver and spleen via systemic leakage $(16-18,34)$. Consistent with the notion that CSF-mediated glymphatic clearance removes solutes from the CNS, we observed a profound decrease in vector uptake and gene expression in off-target organs, such as the heart and liver, following intra-CSF administration in AQP4 ${ }^{-1-}$ mice. Although comprehensive analysis will be required to elucidate the details of AAV transport within the brain, we would like to note that there is no conclusive evidence in the literature or from our observations to suggest that AAV particles physically traverse the AQP4 pore. We believe that AQP4 channels located on astroglial end feet that line paravascular compartments move solutes such as AAV by generating water currents in the brain. To our knowledge, this is the first demonstration that AQP4-mediated CSF turnover drives parenchymal spread, CNS retention, and systemic drainage of AAV vectors. We postulate that assessment of kinetic biomarkers of CSF flux in patients (35) could help identify prospective cohorts, explain variable treatment outcomes, and formulate the optimal combination of AAV capsid, dosage, and infusion parameters to achieve therapeutic efficacy in treating CNS disorders. 


\section{Methods}

Recombinant $A A V$ vector production. An updated triple plasmid transfection protocol was used to generate recombinant AAV9 vectors $(36,37)$. All plasmids used for AAV production in this study were obtained from The University of North Carolina at Chapel Hill vector core. Specifically, HEK 293 cells were transfected with a mixture of (a) the pXR9 helper plasmid; (b) the adenoviral helper plasmid pXX6-80; and (c) pTR-CBh-ScGFP or pTR-CBA-Luc plasmids encoding the GFP or luciferase (Luc) reporter genes driven by the chicken $\beta$ hybrid (CBh) or chicken $\beta$ actin (CBA) promoter and flanked by inverted terminal repeats (ITRs) derived from the AAV2 genome. Iodixanol gradient ultracentrifugation was used to purify AAV vectors. Fluorophore-labeled AAV vectors were generated using an Alexa 647 labeling kit (Thermofisher-A20006) by following the protocol provided by the kit manufacturer. Following fluorophore labeling, the AAV vectors were dialyzed against $1 \times$ PBS for 2 overnight cycles using a 12- to $14-\mathrm{kDa}$ MWCO dialysis column (Millipore 71505-3). Finally, quantitative PCR (QPCR) (Lightcycler 480, Roche Applied Sciences) was used to calculate vg titers with primers designed to selectively bind AAV2 ITRs (forward, 5'-AACATGCTACGCAGAGAGGGAGTGG-3'; reverse, 5'-CATGAGACAAGGAACCCCTAGTGATGGAG-3') (IDT Technologies).

Animal experiments. The constitutive AQP4 ${ }^{-/-}$mouse model (15) was provided by Alan Verkman (UCSF, San Francisco, California, USA). All animal experiments reported in this study were conducted on $\mathrm{AQP}^{-/-}$or $\mathrm{C} 57 / \mathrm{B} 16$ mice.

Intracranial AAV injections. P0 mouse pups were rapidly anesthetized on ice for 2 minutes followed by i.c.v. or intrastriatal (ISTR) injections using a stereotaxic apparatus. AAV vectors $(<3 \mu 1$ total volume for i.c.v. and $<1 \mu$ total volume for ISTR) packaging the CBA-Luc or CBh-ScGFP transgene cassette were injected into the left LV or the striatum with a Hamilton 700 series syringe and 26s gauge needle (Sigma-A1drich) attached to a KOPF-900 small animal stereotaxic instrument (KOPF instruments). Neonatal mouse i.c.v. injections were performed at the following stereotaxic coordinates: $0.5 \mathrm{~mm}$ relative to the sagittal sinus, $2 \mathrm{~mm}$ rostral to transverse sinus, and $1.5 \mathrm{~mm}$ deep. For ISTR administration in neonatal mice, the coordinates were modified to $1.25 \mathrm{~mm}$ relative to the sagittal sinus, $2 \mathrm{~mm}$ rostral to transverse sinus, and $1.5 \mathrm{~mm}$ deep. Mice were revived under a heat lamp and rubbed in the bedding after AAV injections before being placed back with the dam. i.c.v. injections in adult mice were performed using a 33-gauge needle (Sigma-Aldrich) at $0.7 \mathrm{~mm}$ caudal, $1 \mathrm{~mm}$ lateral, and $2 \mathrm{~mm}$ ventral to bregma. The mouse brains were harvested, post-fixed, vibratome sectioned (VT 1200 S, Leica Biosystems), and immunostained 2 weeks after vector administrations (P14). The immunostaining procedure has been described in detail below. For visualizing AAV spread within the mouse brains, Alexa 647-tagged AAV vectors were mixed with $0.5 \%$ dextran $(10-\mathrm{kDa}$ soluble tracer) $(5 \mathrm{mg} / \mathrm{ml})$ (Thermofisher D22910) reconstituted in $1 \times$ PBS prior to i.c.v. injections. 45 minutes later, injected mouse pups were rapidly anesthetized using hypothermic shock and perfused with $1 \times$ PBS followed by $4 \%$ PFA.

$V g$ biodistribution. Neonatal mouse cohorts used for biodistribution studies were sacrificed 3 days after vector administration via the i.c.v. route. Juvenile and aged mice were sacrificed 45 minutes after vector administration for similar analyses. The genomic DNA was extracted from the tissue lysates using the DNeasy kit (Qiagen). Viral genome copy numbers were calculated by performing QPCR (Lightcycler 480, Roche Applied Sciences) using primers specific to luciferase transgene (5'-AAAAGCACTCTGATTGACAAATAC-3' and 5'-CCTTCGCTTCAAAAAATGGAAC-3') or GFP transgene (5'-AGCAGCACGACTTCTTCAAGTCC-3' and 5'-TGTAGTTGTACTCCAGCTTGTGCC-3'). The vg copy numbers were normalized to mouse lamin B2 locus as the housekeeping gene using the primers 5'-GGACCCAAGGACTACCTCAAGGG-3' and 5'-AGGGCACCTCCATCTCGGAAAC-3'. Vector biodistribution is represented as the ratio of vg per cell recovered in the peripheral organs to the CNS site of injection (brain).

Tissue processing and immunostaining. Mouse cohorts used for gene expression (GFP) studies were sacrificed 2 weeks after vector administration via i.c.v. route. Mouse cohorts used for visualizing viral spread were sacrificed 45 minutes after fluorescent AAV injections. The mice were overdosed with tribromoethanol (avertin) (0.2 ml/10 g of $1.25 \%$ solution) via the intraperitoneal route. This was followed by transcardial perfusions of PBS and 4\% PFA in $1 \times$ PBS. The organs were removed and post-fixed for 24 hours prior to sectioning. Briefly, 50- $\mu \mathrm{m}$-thick sections were obtained using a Leica VT $1200 \mathrm{~S}$ vibrating blade microtome (Leica Biosystems). The sections of mouse organs from various treatments were blocked in $10 \%$ goat serum (Sigma-Aldrich) and 1\% Triton X (Sigma-Aldrich) in PBS for 1 hour. This was followed by overnight incubation with primary monoclonal antibodies at $4^{\circ} \mathrm{C}$. The primary antibodies utilized as a part 
of this study are as follows: rabbit anti-GFP (Life Technologies G10362, 1:750), rabbit anti-AQP4 (Millipore $\mathrm{AB} 3594,1: 750$ ). Secondary antibodies were raised in goats and conjugated to Alexa 488 (anti-rabbit Abcam 96883) or Alexa 594 (anti-rabbit Abcam 96885). The secondary antibodies were used at a standard dilution of 1:500. The immunohistochemical analyses of GFP and AQP4 expression were conducted using the Vectastain ABC kit (Rabbit IgG PK-4001 kit, Vector biolabs).

Microscopy and image processing. We used a Zeiss CLSM 700 confocal laser scanning microscope (Microscopy Services Laboratory, The University of North Carolina at Chapel Hill) or an Aperio Slide scanner (Leica Biosystems) (Translational Pathology Laboratory, The University of North Carolina at Chapel Hill) for imaging immunostained mouse tissue sections. Quantitation of fluorescence/pixel intensities was carried out using the ImageJ image analysis software (NIH). For calculating AAV/dextran fluorescence, the mean pixel intensity function was applied across identical regions of interest and threshold settings in specific anatomical regions of the mouse brain. For calculating perivascular AQP4 polarity, fluorescence intensity values were recorded at 50 different pixel positions across 4 to 5 major cortical blood vessels in the mouse brain. The procedure for quantitation of perivascular AQP4 polarity was adopted from a previous study (14). Confocal images were stitched, pseudocolored, and analyzed on the Zen Black software.

Statistics. Comparisons between 2 groups were determined by 2-tailed Student's $t$ test. $P$ values of less than 0.05 were considered significant.

Study approval. All animal experiments reported in this study were conducted on AQP4 ${ }^{-/}$or C57/ B16 mice bred and maintained in accordance to NIH guidelines, as approved by the The University of North Carolina at Chapel Hill Institutional Animal Care and Use Committee protocol 15-109.

\section{Author contributions}

GM and AA conceived the study. GM carried out vector production, neonatal intracranial AAV administrations, histology, and image analysis. RAR assisted with the AAV biodistribution studies. AC and JS assisted with adult intracranial AAV administrations and provided input for the overall study. GM and AA analyzed the data and wrote the manuscript.

\section{Acknowledgments}

We would like to thank Alan Verkman (UCSF) for providing us with the AQP4 ${ }^{-/-}$mouse strain and the NIH for research funding awarded to AA (R01HL089221; P01HL112761; P30AI027767-24).

Address correspondence to: Aravind Asokan, CB \# 7352, Gene Therapy Center, 5123 Thurston Building, The University of North Carolina at Chapel Hill, Chapel Hill, North Carolina 27599-7352, USA. Phone: 919.843.7621; E-mail: aravind@med.unc.edu.

1. Tuszynski MH, et al. Nerve growth factor gene therapy: activation of neuronal responses in Alzheimer disease. JAMA Neurol. 2015;72(10):1139-1147.

2. Bartus RT, Weinberg MS, Samulski RJ. Parkinson's disease gene therapy: success by design meets failure by efficacy. Mol Ther. 2014;22(3):487-497.

3. Samaranch L, et al. Slow AAV2 clearance from the brain of nonhuman primates and anti-capsid immune response. Gene Ther. 2016;23(4):393-398.

4. Iliff JJ, Nedergaard M. Is there a cerebral lymphatic system? Stroke. 2013;44(6 suppl 1):S93-S95.

5. Abbott NJ. Evidence for bulk flow of brain interstitial fluid: significance for physiology and pathology. Neurochem Int. 2004;45(4):545-552.

6. Cserr HF, Harling-Berg CJ, Knopf PM. Drainage of brain extracellular fluid into blood and deep cervical lymph and its immunological significance. Brain Pathol. 1992;2(4):269-276.

7. Koh L, Zakharov A, Johnston M. Integration of the subarachnoid space and lymphatics: is it time to embrace a new concept of cerebrospinal fluid absorption?. Cerebrospinal Fluid Res. 2005;2:6.

8. Iliff JJ, et al. A paravascular pathway facilitates CSF flow through the brain parenchyma and the clearance of interstitial solutes, including amyloid $\beta$. Sci Transl Med. 2012;4(147):147ra111.

9. Iliff JJ, Goldman SA, Nedergaard M. Implications of the discovery of brain lymphatic pathways. Lancet Neurol. 2015;14(10):977-979.

10. Iliff JJ, et al. Impairment of glymphatic pathway function promotes tau pathology after traumatic brain injury. $J$ Neurosci. 2014;34(49):16180-16193.

11. Huang LY, Halder S, Agbandje-McKenna M. Parvovirus glycan interactions. Curr Opin Virol. 2014;7:108-118.

12. Murlidharan G, Samulski RJ, Asokan A. Biology of adeno-associated viral vectors in the central nervous system. Front Mol Neu- 
rosci. $2014 ; 7: 76$.

13. Hoshi A, et al. Characteristics of aquaporin expression surrounding senile plaques and cerebral amyloid angiopathy in Alzheimer disease. J Neuropathol Exp Neurol. 2012;71(8):750-759.

14. Kress BT, et al. Impairment of paravascular clearance pathways in the aging brain. Ann Neurol. 2014;76(6):845-861.

15. Ma T, Yang B, Gillespie A, Carlson EJ, Epstein CJ, Verkman AS. Generation and phenotype of a transgenic knockout mouse lacking the mercurial-insensitive water channel aquaporin-4. J Clin Invest. 1997;100(5):957-962.

16. Gray SJ, Matagne V, Bachaboina L, Yadav S, Ojeda SR, Samulski RJ. Preclinical differences of intravascular AAV9 delivery to neurons and glia: a comparative study of adult mice and nonhuman primates. Mol Ther. 2011;19(6):1058-1069.

17. Xie J, et al. MicroRNA-regulated, systemically delivered rAAV9: a step closer to CNS-restricted transgene expression. Mol Ther. 2011;19(3):526-535.

18. Yang B, et al. Global CNS transduction of adult mice by intravenously delivered rAAVrh. 8 and rAAVrh. 10 and nonhuman primates by rAAVrh.10. Mol Ther. 2014;22(7):1299-1309.

19. Gray SJ. Gene therapy and neurodevelopmental disorders. Neuropharmacology. 2013;68:136-142.

20. Simonato M, Bennett J, Boulis NM, et al. Progress in gene therapy for neurological disorders. Nat Rev Neurol. 2013;9(5):277-291 .

21. Mawuenyega KG, et al. Decreased clearance of CNS beta-amyloid in Alzheimer's disease. Science. 2010;330(6012):1774.

22. Ross CA, Poirier MA. Protein aggregation and neurodegenerative disease. Nat Med. 2004;10(supp1):S10-S17.

23. Lindsay J, et al. Risk factors for Alzheimer's disease: a prospective analysis from the Canadian Study of Health and Aging. Am $J$ Epidemiol. 2002;156(5):445-453.

24. Smith DH, Johnson VE, Stewart W. Chronic neuropathologies of single and repetitive TBI: substrates of dementia? Nat Rev Neurol. 2013;9(4):211-221.

25. May C, Kaye JA, Atack JR, Schapiro MB, Friedland RP, Rapoport SI. Cerebrospinal fluid production is reduced in healthy aging. Neurology. 1990;40(3 pt 1):500-503.

26. Guo Z, et al. Head injury and the risk of AD in the MIRAGE study. Neurology. 2000;54(6):1316-1323.

27. Plassman BL, et al. Documented head injury in early adulthood and risk of Alzheimer's disease and other dementias. Neurology. 2000;55(8):1158-1166

28. Moretti L, Cristofori I, Weaver SM, Chau A, Portelli JN, Grafman J. Cognitive decline in older adults with a history of traumatic brain injury. Lancet Neurol. 2012;11(12):1103-1112.

29. Johnson VE, Stewart W, Smith DH. Widespread $\tau$ and amyloid- $\beta$ pathology many years after a single traumatic brain injury in humans. Brain Pathol. 2012;22(2):142-149.

30. Masamizu Y, et al. Local and retrograde gene transfer into primate neuronal pathways via adeno-associated virus serotype 8 and 9. Neuroscience. 2011;193:249-258.

31. Löw K, Aebischer P, Schneider BL. Direct and retrograde transduction of nigral neurons with AAV6, 8, and 9 and intraneuronal persistence of viral particles. Hum Gene Ther. 2013;24(6):613-629.

32. Castle MJ, Gershenson ZT, Giles AR, Holzbaur EL, Wolfe JH. Adeno-associated virus serotypes 1, 8, and 9 share conserved mechanisms for anterograde and retrograde axonal transport. Hum Gene Ther. 2014;25(8):705-720.

33. Castle MJ, Perlson E, Holzbaur EL, Wolfe JH. Long-distance axonal transport of AAV9 is driven by dynein and kinesin-2 and is trafficked in a highly motile Rab7-positive compartment. Mol Ther. 2014;22(3):554-566.

34. Rosenberg JB, et al. Comparative efficacy and safety of multiple routes of direct CNS administration of adeno-associated virus gene transfer vector serotype rh.10 expressing the human arylsulfatase A cDNA to nonhuman primates. Hum Gene Ther Clin Dev. 2014;25(3):164-177.

35. Fanara P, et al. Cerebrospinal fluid-based kinetic biomarkers of axonal transport in monitoring neurodegeneration. J Clin Invest. 2012;122(9):3159-3169.

36. Pulicherla N, et al. Engineering liver-detargeted AAV9 vectors for cardiac and musculoskeletal gene transfer. Mol Ther. 2011;19(6):1070-1078

37. Shen $\mathrm{S}$, et al. Engraftment of a galactose receptor footprint onto adeno-associated viral capsids improves transduction efficiency. J Biol Chem. 2013;288(40):28814-28823. 